\title{
Thousands of Milky Ways: galaxy satellites and building blocks
}

\author{
Nelson Padilla ${ }^{1}$, Claudia $\operatorname{Lagos}^{1}$ \& Sofía Cora ${ }^{2,3}$ \\ ${ }^{1}$ Departamento de Astronomía y Astrofísica, Universidad Católica de Chile \\ Vicuña Mackenna 4860, Santiago, Chile \\ email: npadilla@astro.puc.cl \\ ${ }^{2}$ Facultad de Ciencias Astronómicas y Geofísicas de la Universidad Nacional de La Plata, \\ and Instituto de Astrofísica de La Plata \\ (CCT La Plata, CONICET, UNLP), Observatorio Astronómico, Paseo del Bosque S/N, 1900 \\ La Plata, Argentina \\ ${ }^{3}$ Consejo Nacional de Investigaciones Científicas y Técnicas, Rivadavia 1917, Buenos Aires, \\ Argentina
}

\begin{abstract}
A semi-analytic model of galaxy formation with and without active galactic nuclei feedback is used to study the nature of possible building blocks (BBs) of $z=0$ galaxies, including those of Milky-Way types. We find that BBs can show an important range of properties arising from environmental variables such as host halo mass, and whether a galaxy is a satellite within its host halo; the stellar formation histories are comparatively faster and the chemical enrichment is more efficient in BBs than in surviving satellites, in accordance with recent metallicity measurements for the Milky Way. These results can be used in combination with observational constraints to continue probing the ability of the cold dark-matter scenario to reproduce the history of galaxy demography in the Universe.
\end{abstract}

Keywords. methods: N-body simulations, Galaxy: evolution, Galaxy: formation, Galaxy: halo, galaxies: evolution, galaxies: formation

\section{Introduction}

The study of the Milky Way (MW) halo and its satellites has allowed the construction of models for the formation of the Galaxy (e.g. Searle \& Zinn 1978). This has become more feasible taking into account the current consensus on the scenario where the stellar halo is actually a collection of stars from the tidal disruption of satellites accreted onto the MW (Igata, Gilmore \& Irwin 1994), and the large amount of data on metallicities of halo stars and MW satellites gathered during the past several years (see a compilation by Geisler et al. 2007).

The current data shows that the metallicities of the MW satellites do not resemble that of the halo stars, indicating that the building blocks (BBs) of the MW were probably different from the present-day satellites. Therefore, any successful model of galaxy formation will need to fulfill this observational result, that there should be an evolution in the properties of satellites of a given galaxy.

There are other observational results that also point in the same direction, for instance the fact that the stellar mass function shows an evolution with redshift (Drory et al. 2005) would indicate that the population of galaxies evolved with time, also in agreement with the change in the average stellar formation rate (SFR) in the Universe (e.g. Hopkins et al. 2005), or the different formation epochs of galaxies of different masses, where low mass objects seem to have formed only recently, and large galaxies started their collapse at much earlier epochs (e.g. Cowie, Songaila \& Cohen 1996). 


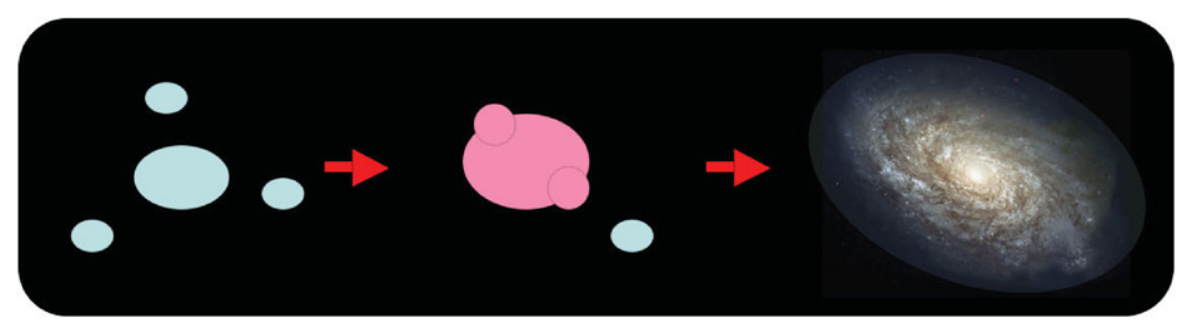

Figure 1. Toy model of galaxy formation. As time increases from left to right, the larger object becomes older and accretes the satellites that formed earlier, all becoming redder (middle figure) except for the newly formed satellites which are blue. The final result is a large spiral galaxy as the one shown on the right.

In this talk we review a recent work by Lagos, Padilla \& Cora (2009, which builds upon work by Bullock \& Johnston 2005, among others), where the process of satellite accretion by central galaxies is followed in the semi-analytic model of galaxy formation by Lagos, Cora \& Padilla (2008). The main assumption for this work consists in that the BBs characterize the stellar content of the halo. This study allows a direct comparison between observations and a $\Lambda$ CDM hierarchical model of galaxy formation, and in particular studies the possibility that the population of satellite galaxies evolves with time in the model.

\section{The formation of a galaxy and its stellar halo}

An important aspect of the formation of galaxies and their satellites comes from the growth of inhomogeneities in an expanding universe. As the fluctuations grow proportional to the scale factor to some power, a large inhomogeneity needs to have started its collapse earlier than smaller ones (on average, e.g. Press \& Schechter 1974). Therefore a possible way for the galaxy formation process to progress is the one shown in Figure 1. The central (larger) galaxy is older than its present-day satellites, and the ones that are in the process of being accreted, have formed earlier than the former and are therefore older and possibly more chemically enriched.

In order to check whether this toy model is a good approximation to the expected evolution of galaxies in a cold dark-matter (CDM) universe where the expansion is starting to be dominated by a cosmological constant (a $\Lambda$ CDM universe), we select semi-analytic galaxies which have at least accreted one of its former satellites, and that still preserve at least one satellite at $z=0$. This is done on the output of the semi-analytic model run over a $\Lambda$ CDM N-body simulation characterised by a periodic box of $60 \mathrm{~h}^{-1} \mathrm{Mpc}$, with a resolution of $1.001 \times 10^{9} \mathrm{~h}^{-1} M_{\odot}$ per DM particle. The simulation parameters are consistent with the results from WMAP data (Sanchez et al. 2006). This simulation is complemented using the non-radiative N-body/SPH (Smoothed Particle Hydrodynamics) simulations of galaxy clusters of masses $M=2.14 \times 10^{15} M_{\odot}$ (Dolag et al. 2005) used in Cora et al. (2008), extending our dynamical range and, consequently, providing a better sampling of the high-mass end of the galaxy population. Due to resolution constraints the analysis only includes galaxies with stellar masses above $10^{7} M_{\odot}$; a much higher resolution would be needed in order to compare the models to the recently discovered ultra faint dwarfs (e.g. Simon \& Geha 2007).

We will check our toy model by studying three particular aspects: (i) How fast the stellar mass of BBs and surviving satellites was acquired, (ii) the peak height of the BBs 

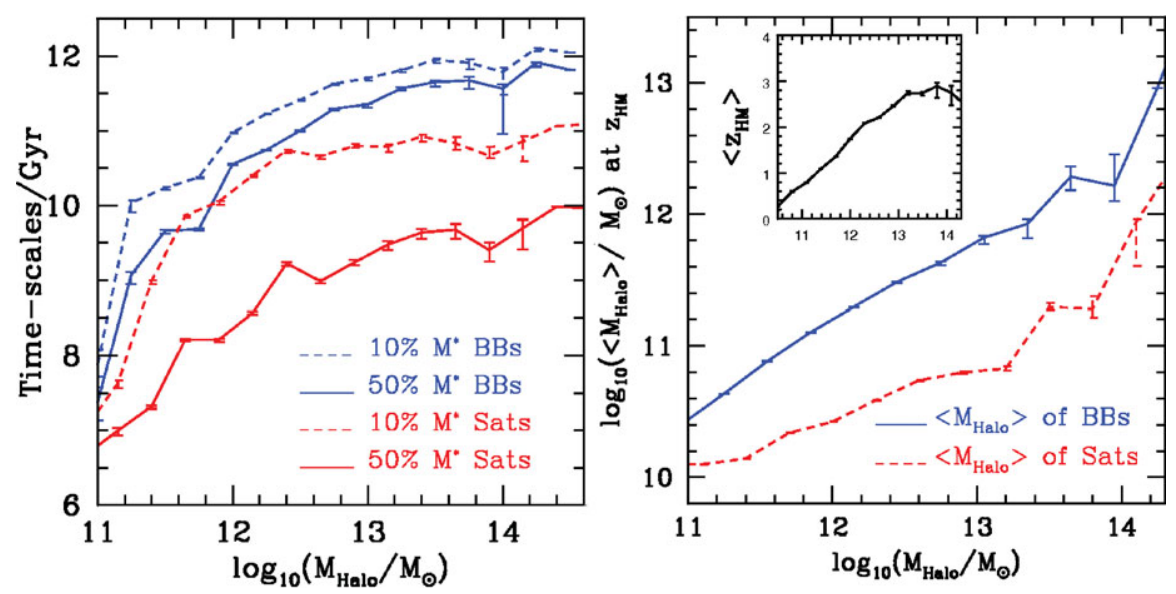

Figure 2. Left panel: Average look-back time (LBT) to the formation of a 10 and 50 per cent of the final stellar mass of BBs and surviving satellites as a function of DM halo mass (line types are indicated in the figure key). Right panel: Central halo mass at the redshift of merger with the parent haloes of BBs and the average mass of parent haloes of surviving satellite galaxies at the same redshift. The average redshift for the merger is shown in the inset. Errorbars show the uncertainty on the mean.

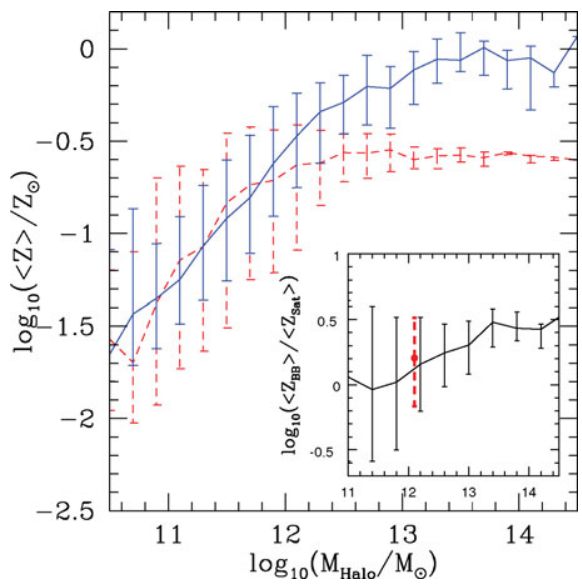

Figure 3. Average metallicity for the present day satellite galaxy population in individual DM haloes (dashed line) and for the BBs of central galaxies (solid line), as a function of the host DM halo mass. Errorbars correspond to the 20 and 80 percentiles. The inset shows the difference between the average metallicities of BBs and satellites as a function of DM halo mass. The filled circle represents MW-type haloes.

and surviving satellites, and (iii) the metallicities of the stars of the BBs before their merger with the central galaxy, and that of the surviving satellites.

For the first point, we simply measure the look-back time to when the satellite or BB had acquired 10 and 50 per cent of its final stellar mass (present-day for satellites, at the moment of disruption for BBs). As a function of dark-matter halo mass, this is shown in the left panel of Figure 2, in blue for BBs (top two lines), and in red for surviving satellites (bottom two lines). As can be seen, regardless of the host dark-matter halo mass, BBs acquire half their final stellar mass approximately four times faster than satellites. This implies that BBs will be characterised by enhanced $\alpha$-element abundance. 
For the second aspect, we will consider that the mass of the host dark-matter halo of the central galaxy at the time of merger with that hosting the $\mathrm{BB}$, and the mass of the dark-matter halo hosting the surviving satellite at the same epoch are good proxies for their relative peak heights. This is shown in the right panel of Figure 2 (the key indicates the meaning of the lines), where as can be seen, the peak height of BBs is indeed higher than that of surviving satellites, confirming that these should have started their collapse earlier. This provides an explanation for the result shown in the left panel, where BBs acquire 10 per cent of their stellar mass about $1 \mathrm{Gyr}$ earlier than surviving satellites.

The third point requires the metallicities of surviving satellites and BBs to be compared. To do this, BBs are studied before they merge with the central galaxy and satellites, at $z=0$. Figure 3 shows the results of this comparison as a function of the host DM halo mass (at $z=0$; satellite results are shown by the dashed line, BBs by the solid line). As can be seen, for hosts of $M>10^{11.8} M_{\odot}$ the metallicities of BBs become higher than that of the surviving satellites. The average metallicity ratio is shown in the inset, and by selecting central galaxies with circular velocities similar to that of the MW (shown by the red dot in the inset) we find that indeed the BBs of MW type galaxies tend to be more metal rich than their $z=0$ satellites.

\section{Outlook}

Within the context of a hierarchical scenario, our study of the formation of the stellar halo in galaxies shows that surviving satellites have different properties than the building blocks of central galaxies, specially for high mass dark-matter haloes. BBs are expected to be more metal rich, to acquire their stellar mass faster, and to have been embedded in higher overdensity peaks than surviving satellites.

In order to ensure the validity of these results, Lagos, Padilla \& Cora (2009) carried out thorough tests on whether the relations between surviving satellites and BBs depend on the model assumptions. They found that switching off active galactic nucleus and supernova feedback produces no appreciable changes. The reason behind this stability of results relies in two facts. On the one hand, BBs form earlier than surviving satellites. On the other, the shaping of the metallicities of low mass objects depends more on the frequency of mergers, which is higher at the redshift of formation of BBs than of surviving satellites (Okamoto et al. 2005), than on the details of the mechanical feedback processes taking place in the evolving galaxy population.

\section{References}

Bullock, J. S., \& Johnston, K. V., 2005, ApJ, 635, 931

Cora, S. A., Tornatore, L., Tozzi, P., \& Dolag, K., 2008, MNRAS, 386, 96

Cowie, L., Songaila, A., Hu, E., \& Cohen, J. G., 1996 AJ, 112, 839

Dolag, K., Vazza, F., Brunetti, G., \& Tormen, G., 2005, MNRAS, 364, 753

Drory, N., Salvato, M., Gabasch, A., Bender, R., Hopp, U., Feulner, G., \& Pannella, M., 2005, ApJ, 619, 131

Geisler D., Wallerstein, G., Smith, V. V., \& Casetti-Dinescu, D. I., 2007, PASP, 119, 939

Hopkins, A., Rao, S., \& Turnshek, D., 2005, ApJ, 630, 108.

Lagos, C., Cora, S., \& Padilla, N., 2008, MNRAS, 388, 587

Okamoto, T., Eke, V. R., Frenk, C. S., \& Jenkins, A., 2005, MNRAS, 363, 1299

Press, W. H., \& Schechter, P., 1974, ApJ, 187, 425

Sanchez, A., et al., 2006, MNRAS, 366, 189.

Searle, L. \& Zinn, R., 1978, ApJ, 225, 357

Simon, J. D., \& Geha, M., 2007, ApJ, 670, 313 